\title{
DIABETES: contribuições sobre prevalência de sintomas gastrointestinais
}

João Paulo Pereira ROSA ${ }^{1}$

\author{
${ }^{1}$ Mestre em Biotecnologia. coord.farmacia@unincor.edu.br
}

Recebido em: 09/05/2016 - Aprovado em: 18/09/2016 - Disponibilizado em: 18/12/2016

\begin{abstract}
RESUMO:
O Diabetes Mellitus é uma doença metabólica crônica, muito frequente em todo o mundo, que pode afetar muitos órgãos e aparelhos. Em decorrência do comprometimento do tubo digestivo, pacientes diabéticos podem apresentar quadros específicos, sendo alguns de grande relevância clínica. Este artigo tem como objetivo demonstrar por meio de estudos, frequências de diferentes sintomas digestivos de pacientes diabéticos. Contribuir para o esclarecimento de dúvidas sobre a questão.

Palavras-chave: Diabetes. Digestivo. Tratamento. Multidisciplinar.
\end{abstract}

\section{DIABETES: contributions of the symptoms prevalence gastrointestinal}

\begin{abstract}
:
Diabetes mellitus is a chronic metabolic disease, very common throughout the world, which can affect many organs and apparatuses. As a result of the digestive tract involvement, diabetic patients may present specific tables, and some of great clinical relevance. This article aims to demonstrate through studies, frequency of digestive symptoms of diabetic patients . Contribute to the clarification of doubts on the issue.
\end{abstract}

Keywords: Diabetes. Digestive. Treatment. Multidisciplinary.

\section{INTRODUÇÃO}

O Diabetes Mellitus é uma doença metabólica crônica, muito frequente em todo o mundo, que pode afetar muitos órgãos e aparelhos. No aparelho digestivo, em particular, é relativamente bem conhecida a ocorrência de complicações do Diabetes Mellitus, como os distúrbios da motilidade do tubo digestivo e as alterações da absorção de água e de eletrólitos, bem como a litíase biliar e a esteatose hepática. Em decorrência do comprometimento do tubo digestivo, pacientes diabéticos podem apresentar quadros específicos, sendo alguns de grande relevância clínica, como gastroparesia diabética e a diarreia do diabético.

O Diabetes Mellitus vem sendo reconhecido hodierna e mundialmente como um problema de saúde pública. São considerados significativos os índices de morbidade e mortalidade relacionados à doença, seu alto custo para a sociedade e os recursos financeiros envolvidos no tratamento e na recuperação de pacientes portadores da patologia. 
No entanto, estudos mais objetivos da prevalência de sintomas digestivos em diabéticos com análise comparativa à frequência encontrada na população geral, não são muito numerosos. Além disso, em pelo menos um destes estudos, feito em amostra expressivamente numerosa, a prevalência de sintomas gastrointestinais nos pacientes diabéticos não foi significativamente diferente da verificada em controles.

É sabido que os sintomas das diversas condições mórbidas e, em especial, os relacionados ao aparelho digestivo, constituem manifestações clínicas subjetivas, que são passíveis de serem influenciadas por fatores externos, como os ligados aos aspectos culturais, à raça, à dieta e ao clima, entre outros. É licito supor que os resultados de estudos sobre prevalência de sintomas em diabéticos realizados no hemisfério Norte possam não ser generalizados para o Brasil onde, ao nosso conhecimento, não existem trabalhos desta natureza.

Um dos itens fundamentais da vida do diabético é o tratamento dietético. A alimentação adequada permite um bom controle metabólico, mas só é possível com a cooperação do paciente.

\section{OBJETIVO}

Demonstrar por meio de estudos, frequências de diferentes sintomas digestivos de pacientes diabéticos. Contribuir para o esclarecimento de dúvidas sobre a questão.

\section{MATERIAL E MÉTODOS}

O trabalho foi realizado por meio de pesquisas em livros, artigos, sites e pesquisas feitas por Universidades de diversos lugares a ocorrência dos Diabetes Mellitus que vem afetando a vida de vários brasileiros hoje em dia por falta de informações.

Buscou-se abordar os principais problemas, dúvidas, importância do tratamento, esclarecendo como é essa doença metabólica crônica.

\section{DESENVOLVIMENTO}

\section{Denomina-se Diabetes mellitus um} grupo heterogêneo de doença que diferem quanto à etiologia e patogênese e que alteram a homeostase do homem, caracterizadas por distúrbios no metabolismo de carboidratos, proteínas e gorduras, secundários a uma deficiência ou ausência de produção de insulina pelo pâncreas e/ou diminuição de sua ação nos tecidos - alvo. Como consequiência surge a hiperglicemia, cuja intensidade tem relação diretamente proporcional à deficiência de insulina (ALMEIDA, 1997).

A hiperglicemia persistente, observada em pacientes mal controlados, ocasiona 
alterações importantes nas membranas basais de capilares sanguíneos com a glicosilação não-enzimática de proteínas e também a ativação da formação de polióis, que se acumulam nas células. Estes fenômenos contribuem para que as chamadas complicações crônicas da doença, ou seja, retinopatia, nefropatia, neuropatia e alterações na microvasculaturaem geral. Estudos realizados nos últimos anos com diabéticos insulinodependentes demonstram que estas complicações podem diminuir quando se realiza o controle rigoroso da glicemia com terapia insulínica intensiva. Os benefícios e riscos deste mesmo esquema terapêutico aplicado a outros diabéticos permanecem obscuros (ALMEIDA, 1997).

A síndrome diabética deve ser enfrentada, portanto, com o combate aos distúrbios metabólicos advindos das descompensações agudas da doença, mas principalmente com a prevenção e o controle das complicações macro e microvasculares, que têm evolução crônica com prevalência crescente e que pioram a qualidade de vida do diabético, são incapacitantes e diminuem sua sobrevida (ALMEIDA, 1997).

\subsection{TIPOS DE DIABETES}

Existem vários tipos de Diabetes Mellitus, como podemos ver a seguir:

\subsubsection{Diabetes Mellitus Tipo I ou Insulino - Dependente}

No diabetes tipo I, a produção de insulina pelo pâncreas é deficiente ou nula. A ausência de insulina determina que o açúcar não possa entrar nas células para ser utilizado na produção de energia. Os tecidos orgânicos são ávidos pela glicose, a qual no diabetes passa a apresentar níveis sangüíneos perigosamente elevados. Este tipo de diabetes inicia-se mais freqüentementena juventude, embora possa aparecer também em adultos de idade avançada. Cerca de 5 a $10 \%$ dos pacientes diabéticos apresentam o diabetes tipo I e necessitam receber o tratamento com insulina (ALMEIDA, 1997). O Diabetes mellitus tipo I surge em geral até os 30 anos, atingindo preferencialmente crianças e adolescentes, podendo entretanto afetar pessoas em qualquer idade. Caracteriza-se por deficiência absoluta de produção de insulina, necessitando do uso exógeno do hormônio de forma definitiva. Em alguns pacientes, nos primeiros meses de doença, pode não haver necessidade do uso de insulina (fase de "luade-mel"), o que ocorrerá inexoravelmente dentro de alguns meses por destruição da reserva pancreática de insulina. Em diabéticos do tipo I, pode-se observar mais comumente (embora não necessariamente) o início abrupto da doença com quadro clínico exuberante. Estes indivíduos em geral são magros ou de peso normal, bastante instáveis, 
sendo difícil o controle metabólico da doença, podendo ocorrer quadros de cetoacidose diabética (ALMEIDA, 1997).

\subsubsection{Diabetes mellitus Tipo II ou Não Insulino - Dependente}

No diabetes tipo II, embora o pâncreas produza insulina, esta encontra-se em quantidade muito baixa ou então ela não é efetiva. Além disso, é possível que os receptores da insulina que controlam $o$ transporte de açúcar para o interior das células não funcione adequadamente ou existam em número insuficiente. $\mathrm{O}$ diabetes tipo II costuma aparecer em indivíduos com mais de 40 anos. A maioria dos pacientes que apresentam o diabetes tipo II recentemente diagnosticados costumam apresentar excesso de peso e podem controlar o diabetes através do emagrecimento ou através de uma dieta. Em certos casos, a fim de atingir o controle da glicose, pode ser necessário lançar mão de uma terapêutica farmacológica por via oral ou então utilizar a insulina na forma injetável (ALMEIDA, 1997). Segundo Almeida (1997), o Diabetes mellitus tipo II cursa com diversos graus de deficiência, na produção e na ação da insulina. Surge, em geral, em indivíduos adultos acima de 40 anos e seu início muito frequentemente é insidioso, podendo levar anos até chegar ao diagnóstico. Cerca de $80 \%$ dos pacientes são obesos, não têm tendência a cetose, sendo portanto estáveis, com menor chance de quadros cetoacidose. Controla-se com dieta adequada, necessitando por vezes do uso de hipoglicemiantes orais, chegando alguns a necessitar do uso complementar de insulina exógena. A sua etiopatogenia é também ligada a fatores genéticos e ambientais. A transmissão familiar deste tipo de diabetes é mais frequente que no tipo I, evidenciando participação genética significativa. Os fatores ambientais são ligados à presença de obesidade (alimentar e diminuição da atividade física). $\mathrm{O}$ desencadeamento da doença muitas vezes ocorre por ocasião do ganho de peso em pessoas geneticamente predispostas. A perda de peso é importante na melhora da tolerância à glicose destes pacientes, favorecendo a atividade insulínica nas células - alvo (ALMEIDA, 1997).

\subsubsection{Diabetes Gestacional}

O diabetes gestacional é aquele surgido ou diagnosticado durante à gestação. Sua importância advém do aumento da morbimortalidade materno - infantil decorrente da associação diabetes e gestação. É muito importante, portanto o controle da doença durante o período pré-natal. Após o parto, a paciente deve ser reclassificada quanto à tolerância glicídica (ALMEIDA, 1997). 


\subsubsection{Outro Tipos de Diabetes}

$\mathrm{O}$ que se denomina outros tipos de diabetes associados a certas síndromes e doenças constitui um grupo heterogêneo de patologias incluindo-se:

- Diabetes pancreático secundário à destruição do órgão;

- Diabetes secundários às endocrinopatias(síndrome de Cushing, acromegalia, etc).

- Diabetes secundários às normalidades de receptores, condições raras de extrema resistência à insulina;

- Diabetes associado às síndromes genéticas como leprechaunismo, ataxia - telangiectasia;

- Atualmente também se estuda um tipo particular de diabetes, característico dos tópicos, associado à desnutrição.

\subsection{A IMPORTÂNCIA DE EQUIPES MULTIDISCIPLINARES NO CURATIVO DO DIABETES MELLITUS TIPO II}

Os objetivos de pesquisa foram pessoas portadoras de diabetes mellitus tipo II, do sexo feminino e masculino, idade acima de 40 anos, aspecto socioeconômico - cultural variável entre aposentados e com renda até 10 salários mínimos. As queixas dos pesquisadores são: falta de informação, falta de recursos para obter informações, ajuda assistencial deficiente, tanto pública como particular e associações filantrópicas(tratamento, econômico, dificuldade de acesso em decorrência da distância, tratamento especifico e quantidade e profissionais deficientes. A reação dos pesquisadores ao saberem da doença revolta, angustia devido à mudança de hábitos (REVISTA UNICASTELO, 2001). Nas complicações do diabetes mellitus tipo II, são mais frequentes a aterosclerose acelerada, e microangiopatias, que podem causar a morte. A fim de entender a necessidade e a natureza do tratamento, o diabético necessita conhecer tudo sobre o processo de sua doença e como enfrentá-la. Por isso o envolvimento de equipes multidisciplinares é essencial para o tratamento utilizado nos curativos. Consequentemente, a interdisciplinaridade dos cuidadores junto aos programas de educação e os cuidado pessoal põem prevenir e melhorar a qualidade de vida das pessoas portadoras de diabetes (REVISTA UNICASTELO, 2001). A lei dos diabéticos traçou novas diretrizes para uma política de prevenção e melhorar a qualidade de vida das pessoas portadoras de diabetes. A lei dos diabéticos traçou novas diretrizes para uma política de prevenção e atenção integral à saúde de portadores da doença, despertando e conscientizando cada indivíduo do seu direito de cidadania. A procura de equipe mais 
próximas da residência para fazer o controle do diabetes é indicada dos portadores. Elas abrangem os multiprofissionais: cirurgião plástico, dentista, dermatológico, educação física, endocrinologista enfermeiro, fisioterapeuta, nefrologista, nutricionista, oftalmologista, ortopedista, pedólogo, psicólogo, técnico ortopédico, urologista e vascular. Existem associações às quais os interessados poderão se associar, como a ANAD - Associação Nacional de Assistência ao Diabético e ADJ - Associação de Diabetes Juvenil (REVISTA UNISCATELO, 2001).

\subsection{NEUROPATIA AUTÔNOMA} CARDIOVASCULAR EM PACIENTES COM DIABETES MELLITUS TIPO II

A prevalência de neuropatia autônoma é muito variável entre os estudos e encontram-se taxas de $17 \%$ a $40 \%$ de anormalidades autonômicas, segundo os testes cardiovasculares de Ewing, que são provas do sistema nervoso autônimo de grande reprodutibilidade e aplicabilidade prática. A ausência de dor em circunstância isquêmicas cardíaca em pacientes com DM tem sido atribuída à presença de NAC ocasionada por alterações na transmissão de impulsos nociceptivos. Foram descritas alterações histopatológicas e diminuição do número de fibras nos nervos do miocárdio de pacientes diabéticos que morreram após IAM sem dor torácica (UNIVERSIDADE FEDERAL DE
SANTA MARIA, 2005). A patogênese exata da neuropatia diabética autônoma permanece incerta. Existem estudos demonstrando o envolvimento de pequenas fibras amielínicas dos sistemas simpático e parassimpático. Estudos em necropsias de pacientes com Diabetes Mellitus tipo I. atingidos por disautonomia grave, mostraram lesões em nível de gânglios simpáticos, do pneumogástrio e dos troncos nervosos simpáticos corroborando estudos anteriores que encontraram estas alterações em animais com diabetes induzidos por estreptomicina (UNIVERSIDADE FEDERAL DE SANTA MARIA, 2005). A neuropatia autônoma diabética caracteriza-se pelo envolvimento de vários órgãos e aparelhos do organismo, simultaneamente ou, não de forma geralmente desigual quanto à intensidade de acometimento e ao tempo de diagnóstico de Diabetes mellitus. A etiologia desta desigualdade não foi claramente definida. Pelo fato desta ampla variação muitas dúvidas permanecem quanto à história natural desse tipo de neuropatia (UNIVERSIDADE FEDERAL DE SANTA MARIA, 2005).

Em pacientes com Diabetes mellitus existe outro tipo de neuropatia, denominada neuropatia periférica, com evolução e história natural bem diferentes da neuropatia autônoma diabética. Assim sendo a ocorrência destes tipos de neuropatia não é simultânea (UNIVERSIDADE FEDERAL DE SANTA MARIA, 2005). 
A melhor terapêutica reside na prevenção. Pacientes diabéticos devem ter um controle glicêmico ótimo para prevenir a ocorrência de NAC, ainda que esta meta exija esforço multidisciplinar. O controle glicêmico ótimo deve ser almejado, inclusive naqueles com NAC já diagnosticada ou com história de isquemia silenciosa. Para detecção de NAC recomendamos a realização das cinco provas de Ewing, relativamente fáceis de serem aplicadas a todos os pacientes diabéticos, sobretudo aqueles com maior duração da doença e os mais idosos. Se estas medidas forem realizadas à NAC, certamente diminuirá. A hipotensão ortostática pode exigir tratamento específico, incluindo suspensão de fatores iatrogênicos, como uso de diuréticos, deve-se recomendar exercícios físicos que melhorem o retorno venoso. Os residentes a essas medidas podem se beneficiar de terapêutica medicamentos com drogas, como as diidroergotaminas e o octreotídeo (UNIVERSIDADE FEDERAL DE SANTA MARIA, 2005).

\subsection{TERAPÊUTICA FARMACOLÓGICA DA DIABETES MELLITUS}

Insulina: é uma molécula proteica, segregada normalmente pelas células beta pancreáticas, que necessita de ser administrada exogenamente em doentes com DID, para equilíbrio metabólico. Sendo destruída pela ação do pH gástrico, não é ativa por administração oral. Atualmente, é utilizada a insulina, designada como humanizadora, pela analógica à insulina natural, no que diz respeito aos aminoácidos que possui e à sua sequência. A insulina sofre modificações gelênicas que lhe alteram os parâmetros farmacocinéticos, permitindo, assim, que se consiga um esquema pesolígico em que a insulina se assemelhe à da produção normal (SOARES, 2001). Só uma formulação se apresenta na forma de solução podendo ser administrada via endovenosa. As suspensões de insulinas são administradas via subcutânea (SC) (SOARES, 2001). As principais diferenças cinéticas entre as insulinas são:

- Velocidade de início de ação

- Tempo para atingir o pico de ação

- Duração de ação

Ocasionadas por alterações gelênicas da molécula, de acordo com estas diferenças, caracterizam-se as insulinas em:

- Ação rápida

- Ação intermédia

- Ação prolongada.

\subsection{FARMACOCINÉTICA (ABSORÇÃO DISTRIBUIÇÃO E ELIMINAÇÃO)}

O início e a duração da ação da insulina é função do tipo de preparação via de administração e de variáveis individuais, como a presença de anticorpos anti-insulina. 
Geralmente, quanto mais elevada é a dose administrada, maior é a intensidade e duração do efeito (SOARES, 2001).

A administração

oral da insulina permite com a administração conjunta de inibidores potentes da alfaquimotripsia, um certo efeito hipoglicemiante, Cho e Flynn mencionaram um sistema oral de libertação de insulina, que utiliza uma microemulsão de água em óleo, contendo insulina a $5 \mathrm{Ul} / \mathrm{ml}$ que, numa dose de $\mathrm{Ul} / \mathrm{Kg}$ (1-3 superior à $\mathrm{SC})$ deu resultados equiparados à insulina SC. Após a administração subcutânea (SC) a insulina é absorvida para a circulação sanguínea, através da via linfática. $O$ passo limitante usa atividade é a velocidade com que sai do local da injeção SC, que é altamente irregular e dependente do tipo de insulina e de outros fatores (ex: o fluxo sangüíneo local e origem - a sintética é absorvida mais rapidamente do que a de origem animal) (SOARES, 2001). Segundo Soares (2001) a insulina encontra-se em forma livre, na corrente sanguínea, a menos que o diabético possua anticorpos circulantes $1 \mathrm{gG}$ para a insulina, aos quais se liga, durante o transporte. A presença destes anticorpos pode demorar ou mascarar o início do efeito da insulina e prolongar a sua semivida biológica. É degradada, em níveis renais $(30-80 \%)$ e extra - renal (fígado e músculo). A sua inativação também ocorre, nos receptores celulares, após a internalização do complexo insulina - receptor. Em nível renal, sofre filtração glomerular e cerca de 99\% do teor filtrado é reabsorvido no tubo proximal e degradado nas células dos capilares glomerulares e nas células dos capilares e nas células penitubularespostglomerulares.

\subsubsection{Locais de injeção}

A insulina é administrada habitualmente por via subcutânea (SC), havendo toda a conveniência de se proceder à rotação do local da injeção, para evitar o desenvolvimento lipodistrofias cutâneas que irão alterar a absorção apesar de se poder proceder a várias injeções, na mesma região do corpo, deve rodar-se o ponto de injeção, para evitar o aparecimento de lipomas (condensações lipídica, no local da injeção, que alteram a velocidade de absorção) e lipoatrofia ( atrofia de gordura com aspecto e depressões cutâneas por desaparecimento lipídico). A velocidade de absorção da insulina, a partir do local da injeção, depende de vários fatores (SOARES 2001).

\subsubsection{Incompatibilidade da Insulina}

Há circunstâncias em que há necessidade de proceder à diluição da insulina, nomeadamente para a perfusão IV, como foi mencionado. Quando diluída nos solutos próprios, a insulina é estável, por períodos longos de tempo (SOARES, 2001).

Emulsões lipidica a 10\%: é 
estável durante 48h, a $4^{\circ} \mathrm{C}$, numa concentração de 160 U/l. Soro Fisiológico: A preparação deve ser administrada de 2-3 horas após a ministrada.

\subsubsection{Contra - Indicações}

Em pessoas alérgicas ao fármaco, situações extremamente raras. Nestes casos, o médico optará por outro tipo de insulina (SOARES, 2001)

\subsubsection{Precauções}

Segundo Soares (2001) Deve haver uma precaução especial com a administração de insulina nos doentes predispostos para a hipoglicemia, nos doentes com deficiência nos mecanismo de contra regulação, nos submetidos a terapêutica com bloqueadores beta adrenérgicos, nos doentes com insuficiência adrenal, pituitária ou autonômica e nos que possuem doença coronária ou cerebrovascular.

Também com os que não aderem corretamente ao tratamento, os alcoólicos ou os que tomam medicamentos psiquiátricos, deve haver precaução com a terapêutica insulina. A administração IV exige uma monotorização mais frequiente, tornandose necessária a administração de potássio. No $2^{\circ}$ e $3^{\circ}$ trimestres da gravidez, as necessidades de insulina podem, estar aumentadas (SOARES, 2001)

\subsubsection{Outros Efeitos}

São normalmente consequência de um mau controle da terapêutica. Por consequência, podem surgir:

- Hiperinsulinemia

- Hipoglicemia

- Hiperglicemia

\subsection{O PAPEL DO FARMACÊUTICO NA ATENÇÃO AO PACIENTE DIABÉTICO}

Portanto, a atenção ao pacientes diabéticos obriga o envolvimento coletivo dos profissionais de saúde nas prevenções primária, secundaria e terciária. $\mathrm{O}$ farmacêutico é o profissional que tem contato diário com um número elevado de pessoas e que é muito procurado para aconselhamento sobre cuidados de saúde. Além disso, em função deste maior contato, tem a possibilidade de receber informações dos pacientes, que podem permitir a suspeita da doença, a avaliação da adesão à terapêutica, da autovigilância e do aparecimento ou agravamento das complicações imediatas ou tardias, podendo consequentemente, colaborar com a equipe de saúde, através dos pacientes, além de poder prestar serviços especializados de atenção farmacêutica (PORTUGAL, 1996) Sendo assim, o papel do farmacêutico no 
diabetes pode ser desenvolvido nos seguintes níveis:

\subsubsection{Prevenção Primária}

O farmacêutico pode contribuir com a prevenção primária através de aconselhamentos da população e pacientes para adoção de estilos de vida saudáveis, principalmente no caso de pessoas em risco de se tornarem diabéticas, através de orientação sobre alimentação saudável, exercício físico, controle de peso, abandono do cigarro e controle da ingestão de álcool (PORTUGAL, 1996).

\subsubsection{Prevenção Secundaria}

O farmacêutico pode participar da prevenção secundaria desenvolvendo as seguintes atividades:

- Aconselhamos os pacientes em risco, para uma atenção cuidadosa aos sinais e sintomas do diabetes e para uma vigilância das suas glicemias tendo em vista a detecção precoce.

- Participação em campanhas de rastreamento regionais ou nacionais, dirigidas a indivíduos em risco para detecção precoce do diabetes e instituições de tratamento adequado pelo médico, após confirmação do diagnóstico.
- Aconselhamos individual dos pacientes da farmácia incluídos em grupos de risco (PORTUGAL, 1996).

\subsubsection{Prevenção Terciária}

No que diz respeito à prevenção das complicações do diabetes, o farmacêutico, como membro integrante da equipe de profissionais de saúde, pode aconselhar pacientes e familiares visando a adesão à terapia farmacológica, não farmacológica, auto - vigilância eautocuidado. Além disso, pode atuar efetuando encaminhamentos a outros profissionais da equipe de saúde, quando da detecção de complicações imediatas e tardias. (PORTUGAL, 1996).

\subsection{MANUSEIO DA DOENÇA RENAL CRÔNICA EM PACIENTES COM HIPERTENSÃO E DIABETES}

A doença renal crônica (DRC), definida como a presença de lesão renal (Proteinúria persistente), associada ou não à diminuição da filtração glomerular $(\mathrm{FG})<$ $60 \mathrm{ml} / \mathrm{mim} / 1,73 \mathrm{~m}^{2}$ por um período $>3$ meses, é um problema de saúde mundial (K/DOQI, 2002). Contudo várias publicações recentes evidenciam que a DRC é subdiagnosticada e subtratada, resultando em evolução desfavorável e alto custo do tratamento (CORESH, 2001). No Brasil, as atenções 
com a DRC se restringem, quase que extremamente, ao seu estágio mais avançado, quando o paciente necessita de terapia de substituição renal (TSR). Contudo a evolução da DRC depende da qualidade do atendimento ofertado muito antes da ocorrência da falência funcional renal (FFR). Embora se reconheçam as vantagens do controle clínico rigoroso nestes pacientes, as instituições das medidas que retardam a progressão da DRC e o tratamento de suas principais complicações e comorbidades estão além do desejado. Muito embora há algum tempo já dispomos de dados sobre a importância do manuseio adequado do diabetes, do controle a pressão arterial, do uso de medicamentos que bloqueiam o sistema renina - angiotensina , da correção da anemia, da acidose metabólica, do tratamento das alterações do cálcio e do fósforo e da necessidade de prevenção das desnutrição, até o momento as informações sobre qualidade do tratamento ambulatorial ofertados aos pacientes com DRC na fase pré - diabética são escassas e geralmente limitadas a estudos internacionais (SOARES et al, 2003).

\subsubsection{Discussão}

O estudo demonstra que os parâmetros clínico - laboratoriais analisados nos prontuários dos pacientes hipertensivos e diabéticos avaliados, considerados de alto risco para desenvolver DRC, não condizem com as recomendações para o controle da doença. As principais razões possíveis para os nossos achados são:

$\checkmark$ A irregularidade na manutenção dos programas de medicamentos gratuitos pelo sistema público.

$\checkmark$ Ainfrequência da realização dos exames laboratoriais necessários para o acompanhamento dos pacientes pelos laboratórios próprios do Sus.

$\checkmark$ Preenchimento inadequado dos prontuários pelos médicos

$\checkmark$ Desconhecimento ou desestimulo da equipe envolvida na implementação das diretrizes sobre controle clínico adequado na DRC.

Esses dados evidenciam que a população estudada, composta de pacientes hipertensivos e diabéticos portadores de DRC e considerada de alto risco para desenvolver falência funcional renal, não está recebendo os cuidados recomendados para o controle clínico de suas doenças. Fica evidente que a gratuidade do atendimento médico somente não garante os cuidados ótimos adequados. Às recomendações atuais, sendo necessário o desenvolvimento de programas de educação continua para os médicos envolvidos no tratamento dos pacientes hipertensivos $\mathrm{e}$ diabéticos e a necessidade de garantia pelo SUS, do suprimento de medicamentos e a da realização de exames laboratoriais em caráter regular (K/DOQI, 2002). 


\section{REFERÊNCIAS BIBLIOGRÁFICAS}

ALMEIDA, Henriqueta GalvaninGuidio.

Diabetes Mellitus Uma Abordagem

Simplificada para Profissionais de Saúde.

São Paulo / Atheneu , 1997. 94 p.

CORESH. J. Wei GL. Mcquillan G.

Prevalence of higt blood pressure and elevated serum creatinine tevei in the unitedStates: Arch Intern Med, 2001. 161p.

K/DOQI, Clinical pratice guidelines for chonic kidney disease evaluation classification and stratification.Am J KidneyDis. $2^{\mathrm{a}}$ ed, 2002. 39 p.

PORTUGAL, Associação Nacional de farmácias. Centro de Documentação e Informação de medicamentos. Programa FarmaDiab: $O$ farmacêutico na Diabetes mellitus. Lisboa : Centro de Documentação, 1996.

SOARES, Maria Augusta. Terapêutica Farmacológica da Diabetes Mellitus. 99a ed :Pharmacia Brasileira, 2001.

SOARES,C.M.B; CANHESTRO, MR et al. Curso clinico da insuficiência renal crônica em crianças e adolescente admitidos no programa interdisciplinar do $\mathrm{HC}$ -

UrMG/MG. J Brás Nefrol, 2003. 25 p.

UNICASTELO, Revista. A importância de equipes multidisciplinares no curativo do diabetes mellitus tipo II. [ S.I. :s.n. ], 2001. p. 62-64. 